\title{
Hamilton saturated hypergraphs of essentially minimum size
}

\author{
Andrzej Ruciński* \\ Department of Discrete Mathematics \\ Adam Mickiewicz University \\ Poznań, Poland \\ rucinski@amu.edu.pl
}

\author{
Andrzej Żak ${ }^{\dagger}$ \\ Faculty of Applied Mathematics \\ AGH University of Science and Technology \\ Kraków, Poland \\ zakandrz@agh.edu.pl
}

Submitted: Jun 28, 2012; Accepted: Apr 29, 2013; Published: May 9, 2013

Mathematics Subject Classifications: 05C65

\begin{abstract}
For $1 \leqslant \ell<k$, an $\ell$-overlapping cycle is a $k$-uniform hypergraph in which, for some cyclic vertex ordering, every edge consists of $k$ consecutive vertices and every two consecutive edges share exactly $\ell$ vertices. A $k$-uniform hypergraph $H$ is $\ell$-Hamiltonian saturated, $1 \leqslant \ell \leqslant k-1$, if $H$ does not contain an $\ell$-overlapping Hamiltonian cycle $C_{n}^{(k)}(\ell)$ but every hypergraph obtained from $H$ by adding one more edge does contain $C_{n}^{(k)}(\ell)$. Let $\operatorname{sat}(n, k, \ell)$ be the smallest number of edges in an $\ell$-Hamiltonian saturated $k$-uniform hypergraph on $n$ vertices. Clark and Entringer proved in 1983 that $\operatorname{sat}(n, 2,1)=\left\lceil\frac{3 n}{2}\right\rceil$. In this paper we prove that $\operatorname{sat}(n, k, \ell)=$ $\Theta\left(n^{\ell}\right)$ for $\ell=1$ as well as for all $k \geqslant 5$ and $\ell \geqslant 0.8 k$.
\end{abstract}

\section{Introduction}

The notion of a hypergraph cycle can be ambiguous. In this paper we are not concerned with the Berge cycles as defined by Berge in [1] (see also [12]). Instead, given integers $1 \leqslant \ell<k$, we define an $\ell$-overlapping cycle as a $k$-uniform hypergraph in which, for some cyclic ordering of its vertices, every edge consists of $k$ consecutive vertices, and every two consecutive edges (in the natural ordering of the edges induced by the ordering of the vertices) share exactly $\ell$ vertices. The notion of an $\ell$-overlapping path is defined similarly. Note that the number of edges of an $\ell$-overlapping cycle with $s$ vertices is $s /(k-\ell)$ (and thus, $s$ is divisible by $k-\ell$ ). The two extreme cases of $\ell=1$ and $\ell=k-1$ are referred

\footnotetext{
*Research supported by the Polish NSC grant N201 604940 and the NSF grant DMS-1102086
}

${ }^{\dagger}$ Research partially supported by the Polish Ministry of Science and Higher Education. 
to as, respectively, loose and tight cycles (paths). We denote an $\ell$-overlapping cycle on $s$ vertices by $C_{s}^{(k)}(\ell)$.

An $\ell$-overlapping Hamiltonian cycle in a $n$-vertex $k$-graph $H$ is any subhypergraph of $H$ isomorphic to $C_{n}^{(k)}(\ell)$. If $H$ contains an $\ell$-overlapping Hamiltonian cycle then $H$ itself is called $\ell$-Hamiltonian. A tight Hamiltonian cycle was introduced in the seminal paper by Katona and Kierstad [16] under the name of a Hamiltonian chain. Since the appearence of [16], $\ell$-Hamiltonian cycles have been studied intensively in the context of Dirac-type properties (for a survey see [17]), Ramsey properties (e.g., in [13, 14]), random hypergraphs $([10,6,7])$. However, the saturation problem for Hamiltonian cycles in hypergraphs is mentioned only in a survey paper by Katona [15].

Given a $k$-uniform hypergraph $H$ (or, shortly, a $k$-graph) and a $k$-element set $e \in H^{c}$, where $H^{c}$ is the complement of $H$, we denote by $H+e$ the hypergraph obtained from $H$ by adding $e$ to its edge set. A $k$-graph $H$ is $\ell$-Hamiltonian saturated, $1 \leqslant \ell \leqslant k-1$, if $H$ is not $\ell$-Hamiltonian but for every $e \in H^{c}$ the $k$-graph $H+e$ is such. The largest number of edges in an $\ell$-Hamiltonian saturated $k$-graph on $n$ vertices, that is, the Turán number for the cycle $C_{n}^{(k)}(\ell)$, denoted by $e x\left(n, C_{n}^{(k)}(\ell)\right)$, has been determined recently in [11]. It turned out that

$$
\operatorname{ex}\left(n, C_{n}^{(k)}(\ell)\right)=\left(\begin{array}{c}
n-1 \\
k
\end{array}\right)+e x(n-1, P),
$$

where $P=P(k, l)$ is the $(k-1)$-uniform, $(\ell-1)$-overlapping path with $\left\lfloor\frac{k}{k-\ell}\right\rfloor$ edges. In particular, for graphs $(k=2)$ the largest size of a Hamiltonian saturated graph is $\left(\begin{array}{c}n-1 \\ 2\end{array}\right)+1$. This value is realized by a unique graph consisting of a clique on $n-1$ vertices and a pedant vertex. Note that this is the only Hamiltonian saturated graph with minimum degree 1 .

In this paper we are interested in the other extreme. For $n$ divisible by $k-\ell$, let $\operatorname{sat}(n, k, \ell)$ be the smallest number of edges in an $\ell$-Hamiltonian saturated $k$-graph on $n$ vertices. In the case of graphs, Clark and Entringer proved in 1983 that $\operatorname{sat}(n, 2,1)=\left\lceil\frac{3 n}{2}\right\rceil$ for $n$ large enough.

For $k$-graphs with $k \geqslant 3$ it seems to be quite hard to obtain such precise results. Therefore, the emphasis is put on the order of magnitude of $\operatorname{sat}(n, k, \ell)$. It was observed in [15] that $\operatorname{sat}(n, k, k-1)=\Omega\left(n^{k-1}\right)$. After some preliminary results in [8,9], the second author showed recently that for $k \geqslant 2$, sat $(n, k, k-1)=\Theta\left(n^{k-1}\right)$, see [18]. Here we extend that result to $\ell$-overlapping Hamiltonian cycles for several other values of $\ell$. Our main result is the following.

Theorem 1.1. For all $k \geqslant 3$ and $\ell=1$, as well as for all $\frac{4}{5} k \leqslant \ell \leqslant k-1$

$$
\operatorname{sat}(n, k, \ell)=\Theta\left(n^{\ell}\right)
$$

We conjecture that Theorem 1.1 holds for all $k$ and $1 \leqslant \ell \leqslant k-1$. 


\section{Preliminaries}

The next two sections contain proofs of the upper bound in Theorem 1.1. Here we give a simple proof of the lower bound.

Proposition 2.1. For all $k \geqslant 2$ and $1 \leqslant \ell \leqslant k-1$

$$
\operatorname{sat}(n, k, \ell)=\Omega\left(n^{\ell}\right) .
$$

Proof. If $H$ is an $\ell$-saturated $k$-graph with $n$ vertices and $m$ edges then for every nonedge $e \in H^{c}$ there is an edge $f \in H$ such that $|e \cap f|=\ell$ (in fact, there are two such edges $f$, since $e$ has to close an $\ell$-overlapping cycle). But for every $f \in H$, the number of $k$-element subsets $e$ which satisfy $|e \cap f|=\ell$ is exactly

$$
\left(\begin{array}{l}
k \\
\ell
\end{array}\right)\left(\begin{array}{l}
n-k \\
k-\ell
\end{array}\right)
$$

Thus, every $f \in H$ can intersect this way at most $\left(\begin{array}{l}k \\ \ell\end{array}\right)\left(\begin{array}{l}n-k \\ k-\ell\end{array}\right)$ nonedges $e$. Hence,

$$
\left(\left(\begin{array}{l}
n \\
k
\end{array}\right)-m\right) \leqslant m \times\left(\begin{array}{l}
k \\
\ell
\end{array}\right)\left(\begin{array}{l}
n-k \\
k-\ell
\end{array}\right)
$$

which implies that $m=\Omega\left(n^{\ell}\right)$.

In the rest of the paper we assume that $G$ is a graph on the vertex set $\{1, \ldots, n\}$. Let $c(G)$ denote the number of components of $G$. Given a subset $T \subseteq V(G)$, let $G[T]$ be the subgraph of $G$ induced by $T$.

Fact 2.2. Let $k, \ell$, and $\Delta$ be constants. If $\Delta(G) \leqslant \Delta$ then the number of $k$-element subsets $T \subseteq V(G)$ with $c(G[T]) \leqslant \ell$ is $O\left(n^{\ell}\right)$.

Proof. The number of $k$-element subsets $T \subseteq V(G)$ with $c(G[T]) \leqslant \ell$ is at most

$$
\left(n(k-1) ! \Delta^{k-1}\right)^{\ell}=O\left(n^{\ell}\right) .
$$

Given a graph $G$ and an integer sequence $\mathbf{a}=\left(a_{1}, \ldots, a_{n}\right)$, the a-blow-up of $G$ is the $k$-graph $H$ with

$$
\begin{aligned}
& V(H)=\bigcup_{i=1}^{n} U_{i}, \quad\left|U_{i}\right|=a_{i}, \\
& H=\bigcup_{i j \in G} K^{(k)}\left(U_{i} \cup U_{j}\right)
\end{aligned}
$$

where $K^{(k)}(U)$ is the complete $k$-graph on $U$ and the sets $U_{i}$ are pairwise disjoint. If $a_{i}=a$ for all $i=1, \ldots, n$, then we simply write $a$-blow-up instead of a-blow-up. For a subset $S \subset V(H)$, let

$$
\operatorname{tr}(S)=\left\{i \in V(G): U_{i} \cap S \neq \emptyset\right\}
$$


Furthermore, set

$$
c(S)=c(G[\operatorname{tr}(S)]) .
$$

The following result is an immediate corollary of Fact 2.2 .

Corollary 2.3. Let $a_{1}, \ldots, a_{n}, k, \ell$, and $\Delta$ be constants. If $\Delta(G) \leqslant \Delta$ and $H$ is an ablow-up of $G$ then the number of $k$-element subsets $S \subseteq V(H)$ with $c(S) \leqslant \ell$ is $O\left(n^{\ell}\right)$.

\subsection{Hamiltonian cycle saturated graphs}

The proofs of the upper bounds in Theorem 1.1 are constructive. The starting points of our constructions are sparse Hamiltonian saturated graphs, also known as maximally non-Hamiltonian graphs. Probably the best known Hamiltonian saturated graphs of minimumm size are Isaac's snarks $J_{k}$ which are 3-regular, connected, bridgeless graphs with chromatic index four, and the number of vertices $n=4 k$. In a series of papers Clark, Crane, Entringer and Shapiro [3, 4, 5] constructed Hamiltonian saturated graphs (by a modification of Isaac's snarks) with minimum possible size for all sufficiently large $n$.

Theorem 2.4 ([5]). For all even $n \geqslant 36$ as well as all odd $n \geqslant 53$ there exists a Hamiltonian saturated graph of order $n$ and size $\lceil 3 n / 2\rceil$.

In order to obtain the right order of magnitude for $\operatorname{sat}(n, k, \ell)$ for all values of $\ell$ considered in the paper, we will need Hamiltonian saturated graphs with bounded maximum degree. (Due to the asymptotic nature of our result, the numerical value of the bound does not matter to us.) By analyzing the construction in [5] one can see that the Hamiltonian saturated graphs obtained there do have bounded maximum degree. An alternative way, which we prefer, is by combining Theorem 2.4 with the following result of Bondy.

Theorem 2.5 ([2]). Let $G$ be a Hamiltonian saturated graph with $n \geqslant 7$ vertices. If for some $0 \leqslant m \leqslant n$ the graph $G$ has $m$ vertices of degree 2 , then $|E(G)| \geqslant(3 n+m) / 2$.

Corollary 2.6. For all $n \geqslant 52$ there exists a Hamiltonian saturated graph $G$ of order $n$ with $\Delta(G) \leqslant 5$.

Proof. By Theorem 2.4 for all $n \geqslant 52$ there exists a Hamiltonian saturated graph $G$ with $n$ vertices and at most $(3 n+1) / 2$ edges. Clearly, $\delta(G) \geqslant 2$. Hence, by Theorem 2.5 , there is at most one vertex of degree 2 in $G$, and, consequently, no vertex of degree greater than 5 .

\section{The loose case $: \ell=1$}

In this Section we prove Theorem 1.1 for $\ell=1$. We begin with a simple lemma.

Lemma 3.1. If a graph $G$ is not Hamiltonian then the $(k-1)$-blow-up $H$ of $G$ is not 1-Hamiltonian. 
Proof. Suppose that $H$ contains a 1-Hamiltonian cycle $C_{H}=\left\{e_{1}, \ldots, e_{n}\right\}$. Define $f$ : $C_{H} \rightarrow G$ by $f\left(e_{s}\right)=\{i, j\} \in G$, where $e_{s} \in H\left[U_{i} \cup U_{j}\right]$. Since $\left|H\left[U_{i} \cup U_{j}\right] \cap C_{H}\right| \leqslant 1$ for all $1 \leqslant i<j \leqslant n$, the mapping $f$ is one-to-one. Furthermore, $C_{G}=\left\{f\left(e_{s}\right), s=1, \ldots, n\right\}$ is a connected, spanning subgraph of $G$. Moreover, $\delta\left(C_{G}\right) \geqslant 2$. Indeed, fix $i \in\{1, \ldots, n\}$, recall that $\left|U_{i}\right|=k-1$, and observe that every subset of $k-1$ vertices of $C_{H}$ intersects at least two edges of $C_{H}$. Thus, $C_{G}$ is a Hamiltonian cycle in $G$, a contradiction.

In view of Proposition 2.1, in order to prove Theorem 1.1 for $\ell=1$ it suffices to construct for every sufficiently large $N$ divisible by $k-1$, a 1-Hamiltonian saturated $k$ graph $H$ with $N$ vertices and $O(N)$ edges. Let $H_{1}$ be a $(k-1)$-blow-up of a Hamiltonian saturated graph $G$ with $n$ vertices, $n=\frac{N}{k-1}$, and $\Delta(G)=O(1)$. (By Corollary $2.6 G$ exists.) By Lemma 3.1, $H_{1}$ is not 1-Hamiltonian and $|V(H)|=N$. Set $V=V\left(H_{1}\right)$ and let

$$
H_{2}=\left\{e \in\left(\begin{array}{l}
V \\
k
\end{array}\right): \operatorname{tr}(e) \text { is a clique in } G\right\} .
$$

Since for every $e \in H_{1}$ the set $\operatorname{tr}(e)$ spans an edge of $G$, we have $H_{1} \subseteq H_{2}$. Finally, let $H$ be a maximal $k$-graph on the vertex set $V$ such that $H_{1} \subseteq H \subseteq H_{2}$ and $H$ is not 1-Hamiltonian. By Corollary 2.3, $|H| \leqslant\left|H_{2}\right|=O(N)$. The following lemma completes the proof of Theorem 1.1 in the case $\ell=1$.

Lemma 3.2. For every $e \in H^{c}, H+e$ is 1-Hamiltonian.

Proof. By the maximality of $H$ we may restrict our attention to only those $e$ for which $\operatorname{tr}(e)$ is not a clique. Fix one pair $\{i, j\} \notin G$ such that $\operatorname{tr}(e) \supset\{i, j\}$. Without loss of generality (w.l.o.g.) we may assume that $i=1$ and $j=2$. Since $G$ is Hamiltonian saturated, $G+\{1,2\}$ has a Hamiltonian cycle containing the edge $\{1,2\}$. Let $C_{G}$ be a Hamiltonian cycle in $G+\{1,2\}$ corresponding, w.l.o.g., to a cyclic ordering $(1, \ldots, n)$. Set $r_{s}=\left|e \cap U_{s}\right|, s=1,2, \ldots, n$. We build a 1 -Hamiltonian cycle $C_{H}=\left\{e_{1}, \ldots, e_{n}\right\}$ in $H$ by 'tracing' $C_{G}$. In doing so, we make sure that the last vertex of each edge $e_{i}$ belongs to the set $U_{i+1}$ and that $U_{i+1} \subseteq e_{1} \cup e_{i} \cup e_{i=1}$.

Formally, we construct $C_{H}$ as follows. (See Fig. 1 for an illustration.)

- Let $e_{1}=e$ and choose $v_{1} \in e_{1} \cap U_{1}$ and $v_{2} \in e_{1} \cap U_{2}$.

- Further, let $e_{2} \in H\left[U_{2} \cup U_{3}\right]$ with $e_{1} \cap e_{2}=\left\{v_{2}\right\}$ and $\left|e_{2} \cap U_{2}\right|=k-r_{2}$. Note that

$$
\left|e_{2} \cap U_{3}\right|=k-\left|e_{2} \cap U_{2}\right|=r_{2} \leqslant k-1-r_{3}=\left|U_{3} \backslash e_{1}\right|
$$

and $U_{2} \subset e_{1} \cup e_{2}$. Choose $v_{3} \in e_{2} \cap U_{3}$.

- Subsequently, for $3 \leqslant t \leqslant n-1$, let $e_{t} \in H\left[U_{t} \cup U_{t+1}\right]$ with $e_{t-1} \cap e_{t}=\left\{v_{t}\right\}, e_{t} \cap e_{1}=\emptyset$, and $\left|e_{t} \cap U_{t}\right|=k-\sum_{s=2}^{t} r_{s}$. Note that

$$
\left|e_{t} \cap U_{t+1}\right|=\sum_{s=2}^{t} r_{s} \leqslant k-1-r_{t+1}=\left|U_{t+1} \backslash e_{1}\right|,
$$

because $\sum_{s=1}^{n} r_{s}=k$ and $r_{1} \geqslant 1$. Moreover, $U_{t} \subset e_{1} \cup e_{t} \cup e_{t+1}$. Set $v_{t+1} \in e_{t} \cap U_{t+1}$. 
- Finally, let $e_{n} \in H\left[U_{n} \cup U_{1}\right]$ with $e_{n-1} \cap e_{n}=\left\{v_{n}\right\},\left|e_{n} \cap U_{n}\right|=k-\sum_{s=2}^{n} r_{s}$, and $e_{n} \backslash U_{n}=\left(U_{1} \backslash e_{1}\right) \cup\left\{v_{1}\right\}$. Note that

$$
\left|e_{n} \cap U_{1}\right|=\sum_{s=2}^{n} r_{s}=k-r_{1}=1+\left|U_{1} \backslash e_{1}\right|
$$

and $U_{1} \subset e_{n} \cup e_{1}$.

Thus, indeed, $C_{H}=\left\{e_{1}, \ldots, e_{n}\right\}$ is a 1-Hamiltonian cycle in $H$.

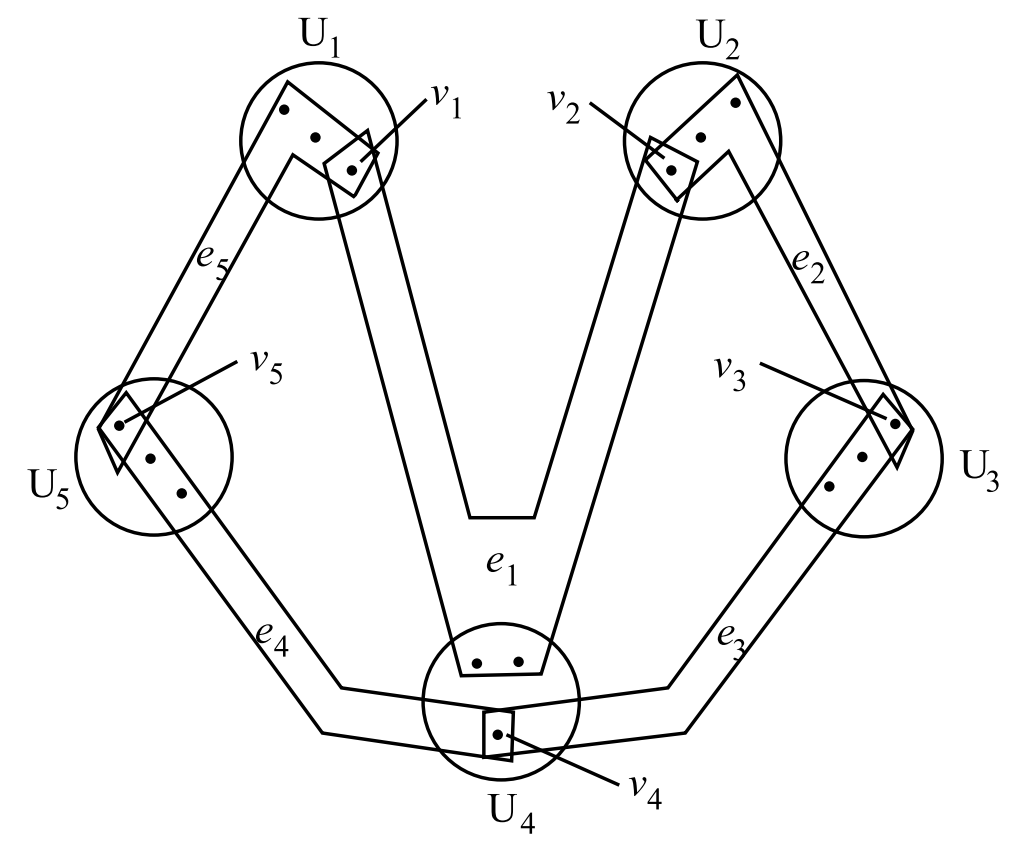

Figure 1: An illustration to the proof of Lemma 3.2: $n=5, k=4$

\section{The case $\ell \geqslant 4 k / 5$}

In this Section we prove our main result, that is, Theorem 1.1 for $\ell \geqslant \frac{4}{5} k$. Let $\mathbf{a}=$ $\left(a_{1}, \ldots, a_{n}\right)$, where

$$
2 k-\ell+1 \leqslant a_{i} \leqslant 4 \ell-2 k+1, \quad i=1, \ldots, n .
$$

Note that under our assumption on $\ell$ we do have $2 k-\ell+1 \leqslant 4 \ell-2 k+1$, and that for all $1 \leqslant \ell \leqslant k-1$

$$
a_{i} \leqslant 2 \ell-1
$$

Let $G$ be an $n$-vertex Hamiltonian saturated graph with $n$ sufficiently large and $\Delta(G)=$ $O(1)$, guaranteed by Corollary 2.6, and let $H_{1}$ be the a-blow-up $k$-graph of $G$ with

$$
V=V\left(H_{1}\right)=\bigcup_{i=1}^{n} U_{i}, \text { where }\left|U_{i}\right|=a_{i}, \quad i=1, \ldots, n .
$$


Observe that for each $e \in H_{1}$, the set $\operatorname{tr}(e)$ is either a vertex or an edge of $G$ and thus $c(e)=1$. Given a set $S \subseteq V$, let

$$
\min (S)=\min \{i: i \in \operatorname{tr}(S)\}=\min \left\{i: U_{i} \cap S \neq \emptyset\right\} .
$$

Further, let $\mathrm{H}_{2}$ be

$$
\left\{e \in\left(\begin{array}{l}
V \\
k
\end{array}\right):\left|e \cap U_{\min (e)}\right| \geqslant k-l+1, c(e) \geqslant k-l+1 \text { and }\left|e \cap U_{\min (e)}\right|+c(e) \geqslant l+2\right\} .
$$

Since for every $e \in H_{2}$ we have $c(e) \geqslant k-\ell+1 \geqslant 2$, the $k$-graphs $H_{1}$ and $H_{2}$ are edge-disjoint.

Lemma 4.1. $H_{1} \cup H_{2}$ is not $\ell$-Hamiltonian.

Proof. Suppose that $H_{1} \cup H_{2}$ contains an $\ell$-Hamiltonian cycle $C_{H}$.

Case 1. Assume first that $C_{H} \subseteq H_{1}$ and define a subgraph $C_{G}$ of $G$ as the set of all 2-element traces $\operatorname{tr}(e)$ of the edges $e$ of $C_{H}$. Formally,

$$
C_{G}=\left\{\operatorname{tr}(e): e \in C_{H} \text { and }|\operatorname{tr}(e)|=2\right\} .
$$

We are going to arrive at a contradiction by showing that $C_{G}$ is a Hamiltonian cycle in $G$. Since, clearly, $C_{G}$ is a connected, spanning subgraph of $G$, it is enough to prove that $C_{G}$ is 2-regular.

Let us fix $i \in\{1, \ldots, n\}$. As $C_{H}$ has to enter and leave the set $U_{i}$ at some point, there exist an edge $e \in C_{H}$ and an index $j \neq i$ such that $\operatorname{tr}(e)=\{i, j\}$. Let $P$ be a longest l-overlapping path in $C_{H}$ (a segment of $C_{H}$ ) containing $e$ and with $\bigcup_{f \in P} \operatorname{tr}(f)=\{i, j\}$. Further, let $e^{\prime}, e^{\prime \prime}$ be the two edges of $C_{H}$ which intersect $V(P)$ each in $\ell$ vertices and set $A^{\prime}=e^{\prime} \cap V(P)$ and $A^{\prime \prime}=e^{\prime \prime} \cap V(P)$ (see Fig. 2). Since on the one hand $\operatorname{tr}\left(A^{\prime}\right) \subseteq\{i, j\}$ while, on the other hand, $A^{\prime} \subset e^{\prime}$ and $\left|\operatorname{tr}\left(e^{\prime}\right) \cap\{i, j\}\right|=1$ (and the same is true for $A^{\prime \prime}$ ) we have $\left|\operatorname{tr}\left(A^{\prime}\right)\right|=\left|\operatorname{tr}\left(A^{\prime \prime}\right)\right|=1$. However, $\operatorname{tr}\left(A^{\prime}\right) \neq \operatorname{tr}\left(A^{\prime \prime}\right)$. Indeed, if, say, $A^{\prime} \cup A^{\prime \prime} \subseteq U_{i}$ then, by (2), we would have $A^{\prime} \cap A^{\prime \prime} \neq \emptyset$ and consequently

$$
e \subseteq V(P) \subseteq A^{\prime} \cup A^{\prime \prime} \subseteq U_{i}
$$

a contradiction with the choice of $e$.

In conclusion, if for some $e \in C_{H}$ we have $\operatorname{tr}(e)=\{i, j\}$ then there is a set $A \subset U_{i}$ with $|A|=\ell$ which on the cycle $C_{H}$ is connected to $e$ by an $\ell$-overlapping path consisting of vertices from $U_{i} \cup U_{j}$ only. Moreover, the edge, say $e^{\prime}$, extending $A$ along $C_{H}$ in the opposite direction (away from $e$ ) satisfies $\operatorname{tr}\left(e^{\prime}\right)=\left\{j^{\prime}, i\right\}$, where $j^{\prime} \neq i, j$, and so $N_{C_{G}}(i) \supseteq\left\{j, j^{\prime}\right\}$. To show that $C_{G}$ is indeed 2-regular, suppose to the contrary that there exist edges $e_{1}, e_{2}, e_{3} \in C_{H}$ with $\operatorname{tr}\left(e_{s}\right)=\left\{i, j_{s}\right\}, s=1,2,3$, where $j_{1}, j_{2}, j_{3}$ are mutually distinct and different from $i$. Let $A_{s}, s=1,2,3$, be the sets described above (with respect to $e_{s}$ ). Since $\left|A_{s}\right|=\ell$, again by (2), the sets $A_{1}, A_{2}, A_{3}$ intersect pairwise. Assume w.l.o.g that $A_{1}$ is located (along $C_{H}$ ) between $e_{1}$ and $e_{2}$. Then $A_{3}$ cannot intersect $A_{1}$, a contradiction. (See Fig. 3 for an illustration of this proof.) 


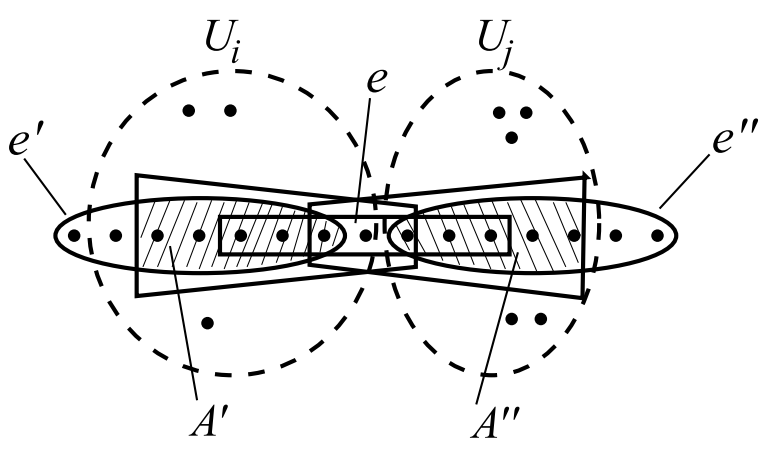

Figure 2: An illustration to the proof of Lemma 4.1: $k=7, \ell=5,\left|U_{i}\right|=\left|U_{j}\right|=10$, the path $P$ consists of 3 "quadrangular" edges.

Case 2. Assume that $C_{H}$ contains a segment of more than $k^{2}$ consecutive edges from $H_{2}$ (that is, an $\ell$-overlapping path in $C_{H} \cap H_{2}$ ). Let $e_{1}, \ldots, e_{s-1}, s>k^{2}+1$, be such a segment. Recall that $\left|e \cap U_{\min (e)}\right| \geqslant k-\ell+1$ for every $e \in H_{2}$, while $\left|e_{t} \cap e_{t+1}\right|=\ell$ for all $t=1, \ldots, s-2$. These two facts imply that $\min \left(e_{t}\right)=\min \left(e_{t+1}\right)$ for $t=1, \ldots, s-2$, and so $\left|e_{t} \cap U_{i}\right| \geqslant k-\ell+1$ for some $i \in[1, n]$ and all $t=1, \ldots, s-1$. On the other hand, observe that a vertex can belong to at most $k$ edges of $C_{H}$. Hence,

$$
\left|U_{i}\right| \geqslant\left|\left(e_{1} \cup \cdots \cup e_{s-1}\right) \cap U_{i}\right| \geqslant \frac{1}{k}(s-1)(k-\ell+1) \geqslant \frac{2}{k}(s-1)>2 k>\left|U_{i}\right|,
$$

a contradiction.

Case 3. Assume that $H_{2} \cap C_{H} \neq \emptyset$ but the longest segment in $C_{H}$ of consecutive edges from $H_{2}$ has length at most $k^{2}$. Let $e_{1}, \ldots, e_{s-1}, 2 \leqslant s \leqslant k^{2}+1$, be such a segment. Then $e_{m} \in H_{1}$ and $e_{s} \in H_{1}$. As in Case 2, $\left|e_{t} \cap U_{i}\right| \geqslant k-\ell+1$ for some $i \in[1, n]$ and all $t=1, \ldots, s-1$. Consequently, $e_{m} \cap U_{i} \neq \emptyset$ as well as $e_{s} \cap U_{i} \neq \emptyset$. By the definition of $H_{1}$, each of $\operatorname{tr}\left(e_{m}\right)$ and $\operatorname{tr}\left(e_{s}\right)$ is either the singleton $\{i\}$ or an edge of $G$ containing vertex $i$ and thus, $c\left(e_{m}\right)=c\left(e_{s}\right)=1$. In view of this and the inequality $c\left(e_{1}\right) \geqslant k-\ell+1$ we have $\left(e_{1} \backslash e_{m}\right) \cap U_{i}=\emptyset$. Analogously, $\left(e_{s-1} \backslash e_{s}\right) \cap U_{i}=\emptyset$. Moreover, in fact $c\left(e_{1}\right)=c\left(e_{s-1}\right)=k-\ell+1$. Therefore, by the third criterion in the definition of $\mathrm{H}_{2}$,

$$
\left|U_{i} \cap\left(e_{1} \cap e_{m}\right)\right|=\left|U_{i} \cap e_{1}\right| \geqslant \ell+2-(k-\ell+1)=2 \ell-k+1
$$

and, similarly,

$$
\left|U_{i} \cap\left(e_{s-1} \cap e_{s}\right)\right| \geqslant 2 \ell-k+1 .
$$

Observe that for large $n, e_{m} \cap e_{s}=\emptyset$. Indeed, if $e_{m} \cap e_{s} \neq \emptyset$, then, necessarily $e_{1} \subseteq e_{s} \cup e_{m}$, and consequently, $c\left(e_{1}\right)=1-$ a contradiction with the definition of $H_{2}$. Hence, we have

$$
\left|U_{i}\right| \geqslant\left|U_{i} \cap\left(e_{1} \cap e_{m}\right)\right|+\left|U_{i} \cap\left(e_{s-1} \cap e_{s}\right)\right| \geqslant 4 \ell-2 k+2,
$$

a contradiction with (1). 


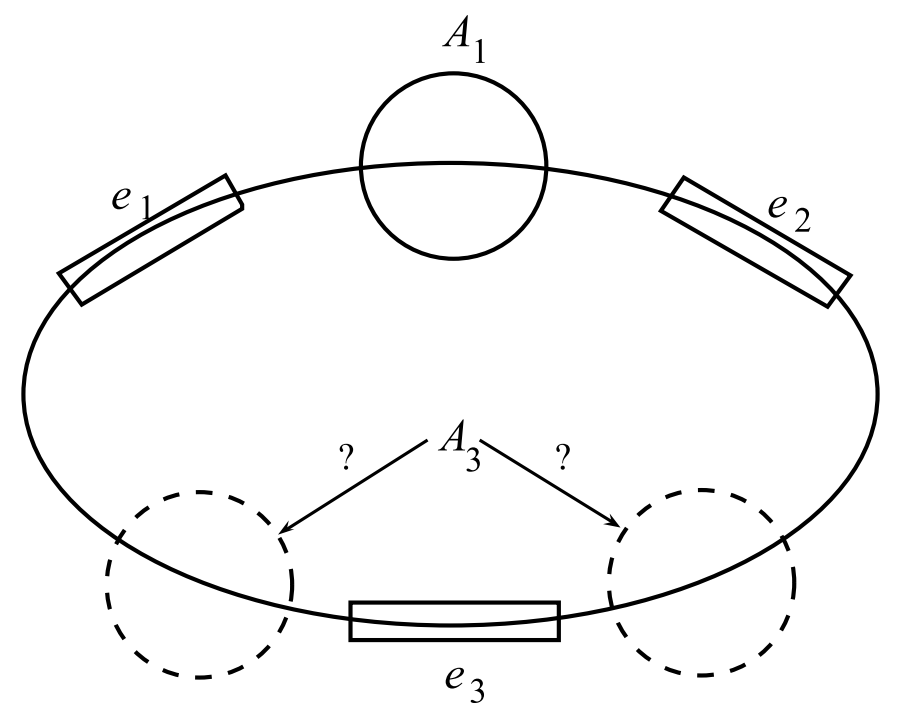

Figure 3: An illustration to the proof of Lemma 4.1

Let

$$
H_{3}=\left\{e \in\left(\begin{array}{l}
V \\
k
\end{array}\right): c(e) \leqslant \ell\right\} .
$$

Recall that for all $e \in H_{1}$ we have $c(e)=1$, while for all $e \in H_{2}$ we have $c(e) \leqslant \ell$. Thus, $H_{1} \cup H_{2} \subseteq H_{3}$. Finally, let $H$ be a maximal $k$-graph on the vertex set $V$ such that $H_{1} \cup H_{2} \subseteq H \subseteq H_{3}$ and $H$ is not $\ell$-Hamiltonian. By Corollary 2.3,

$$
|H| \leqslant\left|H_{3}\right|=O\left(N^{\ell}\right)
$$

We will next show that $H$ is $\ell$-Hamiltonian saturated.

Lemma 4.2. For every $e \in H^{c}, H+e$ is $\ell$-Hamiltonian.

Proof. By the definition of $H$ the thesis holds for each $e$ with $c(e) \leqslant \ell$. Hence, we may assume that $c(e) \geqslant \ell+1$. We will build an $\ell$-overlapping Hamiltonian cycle

$$
C_{H}=\left(e_{1}, \ldots, e_{m}\right)=\left(u_{1}, \ldots, u_{N}\right), \quad m=\frac{N}{k-\ell},
$$

in $H+e$ using the Hamiltonian saturation of $G$. As the general proof is a bit complicated, we will first assume that $\ell=k-1$, in which case the construction can be simplified. This way, avoiding tedious details, we will be able to exhibit the main ideas quite clearly.

The tight case : $\ell=k-1$. We have $k+2 \leqslant a_{j}=\left|U_{j}\right| \leqslant 2 k-3$ for all $j=1, \ldots, n$. Since $c(e) \geqslant k$, the set $\operatorname{tr}(e)$ is, in fact, an independent $k$-element set in $G$. Let

$$
\operatorname{tr}(e)=\left\{i<j_{k-1}<\cdots<j_{1}\right\}
$$


and $e=\left(u_{1}, \ldots, u_{k}\right)$, where $u_{1} \in U_{i}$ and $u_{1+t} \in U_{j_{t}}$ for $t=1, \ldots, k-1$. We construct first a tight path $P \subseteq H_{2}+e$ extending $e$ in both directions, so that the two ends $A$ and $B$ of $P$ are $(k-1)$-tuples contained in, respectively, $U_{i}$ and $U_{j_{k-1}}$. To do so, let $u_{k+t}$, $t=1, \ldots, k-2$, be any vertices of $U_{j_{k-1}}$ different from $u_{k}$, whereas $u_{N-t}, t=0,1, \ldots, k-3$, be any vertices of $U_{i}$ different from $u_{1}$. Then

$$
P=\left(u_{N-k+3}, \ldots, u_{N}, u_{1}, \ldots, u_{2 k-2}\right) .
$$

(See Fig. 4 for an illustration of this construction.)

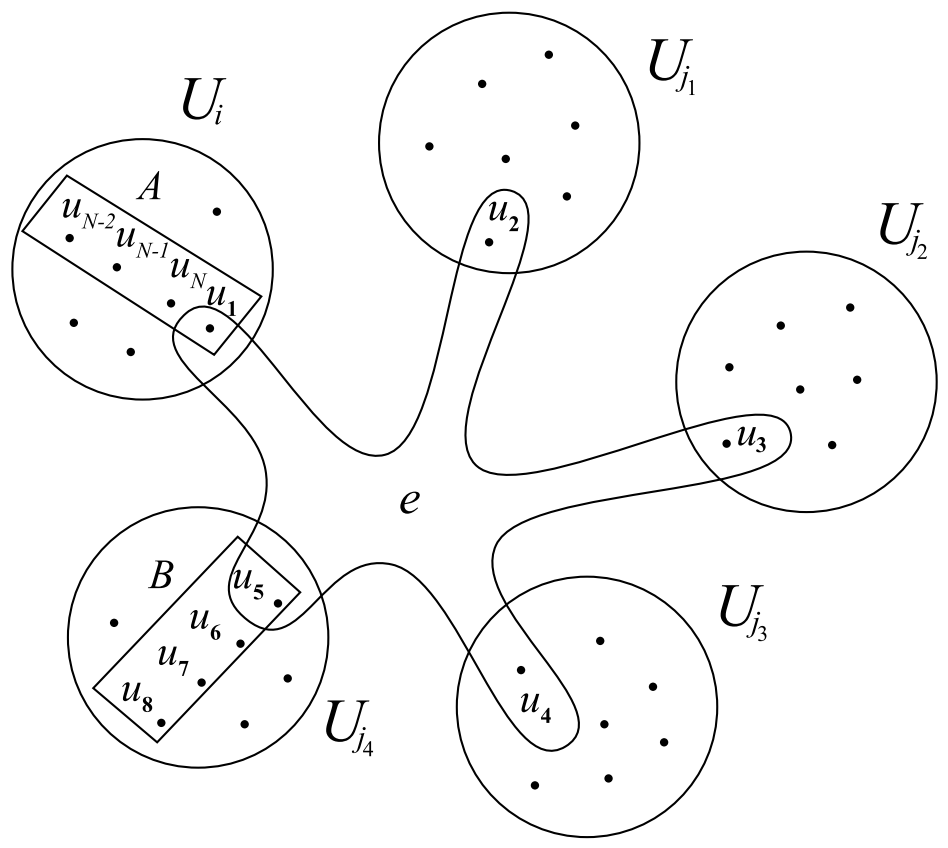

Figure 4: The construction of $P$ in the tight case: $k=5$, all $\left|U_{j}\right|=7$.

To see that $P$ is a tight path in $H+e$ with ends $A=\left(u_{N-k+3}, \ldots, u_{N}, u_{1}\right)$ and $B=\left(u_{k}, \ldots, u_{2 k-2}\right)$, note that for each $q=2, \ldots, k-1$ the edge $e_{q}=\left(u_{q}, \ldots, u_{q+k-1}\right)$ satisfies: $\min \left(e_{q}\right)=j_{k-1},\left|e_{q} \cap U_{j_{k-1}}\right|=q, c\left(e_{q}\right)=k-q+1$, and thus $e_{q} \in H_{2}$. Similarly, for $q=0, \ldots, k-3$, the edges $e_{m-q}=\left(u_{N-q}, \ldots, u_{N}, u_{1}, \ldots, u_{k-q-1}\right)$, for which $\min \left(e_{m-q}\right)=i$, also belong to $\mathrm{H}_{2}$.

Recall that $i j_{k-1} \notin G$ and thus, by the Hamiltonian saturation of $G$ there is a Hamiltonian path $Q$ from $i$ to $j_{k-1}$ in $G$. We connect the ends of $P$, that is, the sets $A$ and $B$, by a tight path $P^{\prime}$ in $H_{1} \subseteq H$, tracing the path $Q$ in $G$ in such a way that every time $Q$ visits a vertex $v$ of $G$ we add to $P^{\prime}$ all vertices of $U_{v}^{\prime}=U_{v} \backslash V(P)$. Since, $\left|U_{v}^{\prime}\right| \geqslant\left|U_{v}\right|-1 \geqslant k-1$ (with some margin), we can always do so by using only the edges of $H_{1}$.

General case. For $\ell \leqslant k-2$ the situation becomes more complicated and the above simple construction of the $\ell$-overlapping path $P$ fails. For instance, if $u_{k-1}$ and $u_{k}$ are in the same component of $G[\operatorname{tr}(e)]$ and $k-2$ is divisible by $k-\ell$, then $c\left(e_{(k-2) /(k-\ell)+1}\right)=1$, 
and so $e_{(k-2) /(k-\ell)+1} \notin H_{2}$. Nevertheless we manage to follow the same idea by slightly modifying the above construction.

Recall that $c(e) \geqslant \ell+1$. Let $j_{1}>j_{2}>\cdots>j_{\ell}>i=\min (e)$ be some $\ell+1$ elements of $\operatorname{tr}(e)$, belonging to different components of $G[\operatorname{tr}(e)]$ and including $i=\min (e)$. Further, let $e_{1}=e=\left(u_{1}, \ldots, u_{k}\right)$, where $u_{1} \in U_{i}$ and $u_{k-\ell+t} \in U_{j_{t}}, t=1, \ldots, \ell$, while $u_{2}, \ldots, u_{k-\ell}$ remain unspecified.

Our plan is, again, first to construct a path $P \subseteq H_{2}+e$ extending $e$ in both directions (Part 1), and then to complete $C_{H}$ by connecting the ends of $P$ by a path $P^{\prime} \subseteq H_{1}$ (Part $2)$. The path $P^{\prime}$ will follow a Hamiltonian path $Q$ in $G$ which together with the pair $\left\{i, j_{\ell}\right\}$ forms a Hamiltonian cycle in $G+\left\{i, j_{\ell}\right\}$.

Part 1. Let integers $q$ and $r$ be defined by

$$
(q+1)(k-\ell)+r=k, \quad 1 \leqslant r \leqslant k-\ell .
$$

The $\ell$-path $P$ will consist of $3 q+5$ edges, $e_{m-2 q}, \ldots, e_{q+4}$, and thus, of $k+(3 q+4)(k-\ell)$ vertices, $u_{N-k+2 r-\ell+1}, \ldots, u_{N}, u_{1}, \ldots, u_{4 k-2 \ell-r}$. The edges are determined by the vertices as they begin at every $(k-\ell)$ th vertex. (Note that $k+\ell-2 r=(2 q+1)(k-\ell)$, and thus $e_{1}$, the $(2 q+2)$ nd edge of $P$ does coincide with $e$.)

We now list all the vertices of $P$, that is, for each index $x$ we specify the set $U_{j}$ from which we (arbitrarily) select a vertex $u_{x}$.

1. For $N-k+2 r-\ell+1 \leqslant x \leqslant N-k+2 r$ we select $u_{x} \in U_{i}$; thus, $P$ begins with $\ell$ vertices of $U_{i}$; we denote their set by $I_{1}$.

2. For $N-k+2 r+1 \leqslant x \leqslant N-k+\ell$ we select $u_{x} \in U_{j_{t}}$, where $t=x-(N-k)-1$; this segment of $P$ has exactly one vertex from each set $U_{j_{2 r+t}}, t=0, \ldots, \ell-2 r-1$; we denote this set by $M_{1}$ ("M" like in mixed).

3. For $N-k+\ell+1 \leqslant x \leqslant N$ we select $u_{x} \in U_{i}$; thus, $P$ returns to $U_{i}$ for $k-\ell$ steps; we denote this set enlarged by $u_{1}$, the first vertex of $e_{1}$, by $I_{2}$.

4. The next $k$ vertices of $P$ are the vertices of $e=e_{1}$, namely $u_{1}, \ldots, u_{k}$; we set $X=\left\{u_{2}, \ldots, u_{k-\ell}\right\}$ and $M_{2}=\left\{u_{k-\ell+1}, \ldots, u_{k-1}\right\}$ (we know nothing about the elements of $X)$.

5. For $k+1 \leqslant x \leqslant 2 k-\ell$ we select $u_{x} \in U_{j_{\ell}}$; thus, $P$ traverses through some $k-\ell$ vertices of $U_{j_{\ell}}$; we denote this set enlarged by $u_{k}$, the last vertex of $e_{1}$, by $L_{1}$.

6. For $2 k-\ell+1 \leqslant x \leqslant 4 k-3 \ell-r$ we select $u_{x} \in U_{j_{t}}$, where $t=x-2 k+\ell$; this segment of $P$ has exactly one vertex from each set $U_{j_{t}}, t=1, \ldots, 2 k-2 \ell-r$; we denote this set by $M_{3}$.

7. For $4 k-3 \ell-r+1 \leqslant x \leqslant 4 k-2 \ell-r$ we select $u_{x} \in U_{j_{\ell}}$; thus, $P$ ends with $\ell$ vertices from $U_{j_{\ell}}$; we denote their set by $L_{2}$. 


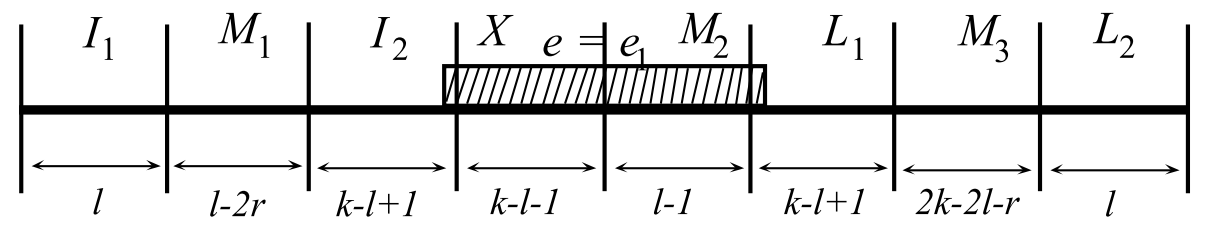

Figure 5: The construction of the $\ell$-path $P$

The construction of the path $P$ is illustrated in Fig. 5 .

Let us now estimate how many vertices of each set $U_{j}$ are used by the above constructed path $P$. Set $r_{j}=\left|V(P) \cap U_{j}\right|, j=1, \ldots, n$.

Fact 4.3. $r_{i} \leqslant 2 k-\ell, r_{j_{\ell}} \leqslant 2 k-\ell$ and $r_{j} \leqslant k-\ell+2$ for all $j \notin\left\{i, j_{\ell}\right\}$.

Proof. Note that since $c\left(e_{1}\right) \geqslant \ell+1,\left|e_{1} \cap U_{j}\right| \leqslant k-\ell$ for each $j \in[1, n]$. In addition, $P$ uses $\ell+(k-\ell)=k$ vertices of both, $U_{i}$ and $U_{j_{\ell}}$, and at most two vertices of each set $U_{j_{t}}$, $t=1, \ldots, \ell-1$.

Fact 4.4. Every edge of $P$ belongs to $\mathrm{H}_{2}+e$.

Proof. Let us split the edges of $P$ into those appearing "before $e$ " (b.e.) and "after $e$ " (a.e.) Formally, set

$$
P=B_{e} \cup\{e\} \cup A_{e},
$$

where $B_{e}=\left\{e_{m-2 q}, \ldots, e_{m}\right\}$ and $A_{e}=\left\{e_{2}, \ldots, e_{q+4}\right\}$ (recall that $e_{1}=e$ ). We will give the proof first for the a.e. edges and then for the b.e. edges. Set

$$
I=I_{1} \cup I_{2} \quad M=M_{1} \cup M_{2} \cup M_{3} \quad L=L_{1} \cup L_{2} .
$$

Let $f \in P-e$.

Case $f \in A_{e}$ : In this case, $f \cap X=\emptyset$ and $\min (f)=j_{\ell}$. Consequently, $f \cap U_{\min (f)}=f \cap L$ and our first goal (c.f. the definition of $\mathrm{H}_{2}$ ) is to show that

$$
|f \cap L| \geqslant k-\ell+1 \text {. }
$$

Observe that either $f \supset L_{1}$, in which case (5) is true, or $f \subset L \cup M_{3}$ and so,

$$
|f \cap L|=k-\left|M_{3}\right|=k-(2 k-2 \ell-r)=2 \ell-k+r \geqslant k-\ell+1,
$$

because $r \geqslant 1$ and $\ell \geqslant \frac{2}{3} k$. Thus (5) holds again.

As a next step we will show that

$$
c(f) \geqslant k-\ell+1 \text {. }
$$

Note that

$$
|f \cap M|=\left|f \cap\left(M_{2} \cup M_{3}\right)\right| \leqslant k-\left|L_{1}\right|=k-(k-\ell+1)=\ell-1 .
$$


Hence, the elements of $f \cap M_{2}$ and $f \cap M_{3}$ come from different sets $U_{j}, j \in\{1, \ldots, \ell-1\}$ and, consequently, $c(f)=|f \cap M|+1$, where we add 1 because of $U_{j_{\ell}}$ (recall that the set $\left\{j_{1} \ldots, j_{\ell}\right\}$ is independent in $\left.G\right)$. If $f \supset M_{3}$ then

$$
c(f) \geqslant\left|M_{3}\right|+1=(2 k-2 \ell-r)+1 \geqslant k-\ell+1,
$$

since $r \leqslant k-\ell$. Otherwise, $f \cap L_{2}=\emptyset$ and

$$
c(f)=k-\left|L_{1}\right|+1=\ell \geqslant k-\ell+1
$$

and (6) holds again. Since, clearly,

$$
\left|f \cap U_{\min (f)}\right|+c(f)=|f|+1=k+1 \geqslant \ell+2,
$$

$f \in H_{2}$, and so $A_{e} \subseteq H_{2}$.

Case $f \in B_{e}$ : In this case, $\min (f)=i$. Consequently, $f \cap U_{\min (f)}=f \cap I$ and our first goal is to show that

$$
|f \cap I| \geqslant k-\ell+1 \text {. }
$$

Observe that the first (that is, with the smallest index) vertex of $f$ coincides with, or is to the left of the first vertex of $I_{2}$. Thus, $f \supset I_{2}$ or

$$
|f \cap I| \geqslant k-\left|M_{1}\right|=k-\ell+2 r \geqslant k-\ell+2,
$$

and in either case (7) holds.

As a next step we will prove that

$$
c(f) \geqslant k-\ell+1 \text {. }
$$

Note that

$$
|f \cap M|=\left|f \cap\left(M_{1} \cup M_{2}\right)\right| \leqslant k-\left|I_{2}\right|-|X|=k-2(k-\ell) \leqslant \ell-1 .
$$

Hence, the elements of $f \cap M_{1}$ and $f \cap M_{2}$ come from different sets $U_{j}, j \in\{1, \ldots, \ell-1\}$ and, again, $c(f)=|f \cap M|+1$. If $f \cap I_{1}=\emptyset$ then

$$
|f \cap M| \geqslant k-\left|I_{2}\right|-|X|=k-2(k-\ell) \geqslant k-\ell,
$$

because $\ell \geqslant \frac{2}{3} k$. If $f \supset M_{1}$ then

$$
|f \cap M| \geqslant\left|M_{1}\right|=\ell-2 r \geqslant k-\ell,
$$

because $r \leqslant k-\ell$ and $\ell \geqslant \frac{3}{4} k$. Otherwise, that is, when $f \cap I_{1} \neq \emptyset$ but $f \not \supset M_{1}$,

$$
|f \cap M|=k-\left|f \cap I_{1}\right| \geqslant k-\ell
$$

Thus, (8) holds in all cases. 
It remains to prove that

$$
\left|f \cap U_{\min (f)}\right|+c(f) \geqslant \ell+2 .
$$

Recall that $\min (f)=i$ and $\left|f \cap U_{i}\right|=|f \cap I|$, while $c(f)=|f \cap M|+1$. Since, clearly,

$$
|f \cap I|+|f \cap M|+|f \cap X|=|f|=k,
$$

we have

$$
\left|f \cap U_{\min (f)}\right|+c(f) \geqslant k+1-|f \cap X| \geqslant k+1-(k-\ell-1)=\ell+2 .
$$

Hence, $f \in H_{2}$ and, consequently, $B_{e} \subseteq H_{2}$. This completes the proof of Fact 4.4.

Part 2. Recall that $\left\{i, j_{l}\right\}$ is not an edge of $G$. Hence, by the Hamiltonian saturation property of $G$, there is a Hamiltonian path $Q$ from $j_{\ell}$ to $i$ in $G$. As in the loose $(\ell=1)$ and tight $(\ell=k-1)$ cases treated earlier, we build the rest of $C_{H}$ by 'tracing' $Q$. Each time we visit a vertex $x \in V(Q)$ we consecutively include to $C_{H}$ all vertices from $U_{x} \backslash V(P)$ (in any order). This way we create an $\ell$-path $P^{\prime}$ consisting of $k$-tuples $e_{q+5}, \ldots, e_{m-2 q-1}$.

Note that by Fact 4.3 and the lower bound in (1), we have

$$
\left|U_{x} \backslash V(P)\right|=\left|U_{x}\right|-r_{x} \geqslant(2 k-\ell+1)-(k-\ell+2)=k-1
$$

for each $x \in V(Q) \backslash\left\{i, j_{\ell}\right\}$. Hence, $\left|\operatorname{tr}\left(e_{j}\right)\right| \leqslant 2$, for all $j=q+5, \ldots, m-2 q+1$. Moreover, for each such $j$ with $\left|\operatorname{tr}\left(e_{j}\right)\right|=2$ the pair $\operatorname{tr}\left(e_{j}\right)$ is an edge of $G$. Therefore, $e_{j} \in H_{1}$, for $j=q+5, \ldots, m-2 q+1$. In conclusion, $C_{H}=P \cup P^{\prime}$ is an $\ell$-Hamiltonian path in $H_{1} \cup H_{2}+e \subseteq H+e$, which completes the proof of Lemma 4.2.

The conclusion of the proof of Theorem 1.1. In order to prove Theorem 1.1 for $\ell \geqslant \frac{4}{5} k$ we need to construct, for every sufficiently large $N$ divisible by $k-\ell$, an $\ell$ Hamiltonian saturated $k$-graph $H$ with $N$ vertices and $O\left(N^{\ell}\right)$ edges. Assume first that $\ell>\frac{4}{5} k$. As then $2 k-\ell+2 \leqslant 4 \ell-2 k+1$ we may use as the sizes $a_{i}=\left|U_{i}\right|$ both numbers, $2 k-\ell+1$ and $2 k-\ell+2$. It is well known that every number $N \geqslant N_{0}=N_{0}(k, \ell)$ (the Frobenius number) can be expressed as a sum of these two numbers. For an $N$ divisible by $k-\ell$, let us fix one such partition

$$
N=a_{1}+\cdots+a_{n}, \quad 2 k-\ell+1 \leqslant a_{i} \leqslant 2 k-\ell+2,
$$

and let $H$ be as in Lemma 4.2. Then, by (3), $H$ indeed is an $\ell$-Hamiltonian saturated $k$-graph with $N$ vertices and $O\left(N^{\ell}\right)$ edges.

In the critical case $\ell=\frac{4}{5} k$, we need to refine our previous estimates a bit. Assume that for some integer $p \geqslant 1$, we have $k=5 p$ and $\ell=4 p$. Then, by (4), $r=p$, and so, $2 r=2 p>2 k-2 \ell-r=p$. Thus, every index $j \in\left\{j_{1}, \ldots, j_{\ell-1}\right\}$ appears at most once in the set $M_{1} \cup M_{3}$, and consequently, we can improve the bound on $r_{j}$ from Fact 4.3 down to $k-\ell+1$. This implies, in turn, that the crucial estimate $\left|U_{x}\right|-r_{x} \geqslant k-1$ from Part 2 of the construction of the cycle $C_{H}$ in the proof of Lemma 4.2 (see (10)) remains valid 
even for sets $U_{x}$ with $\left|U_{x}\right|=2 k-\ell$. Note that the lower bound in (1) was not used in any other part of the proof. We may thus complete the proof as before, expressing $N$ this time as

$$
N=a_{1}+\cdots+a_{n}, \quad 2 k-\ell \leqslant a_{i} \leqslant 2 k-\ell+1 .
$$

\section{$5 \quad$ Remarks and open problems}

Note that in the case $\ell=k-1$ our Theorem 1.1, as stated, covers only $k \geqslant 5$. However, in the proof of Lemma 4.2 we could have $k \leqslant a_{j}=\left|U_{j}\right|=k+1$. Indeed, then we still have $\left|U_{j}^{\prime}\right| \geqslant\left|U_{j}\right|-1 \geqslant k-1$, while the punch-line inequality in the proof of Lemma 4.1, that is, $\left|U_{i}\right| \leqslant k+1 \leqslant 4 \ell-2 k+1=2 k-3$ holds already for $k \geqslant 4$. So, in fact, our proof of Theorem 1.1 works also in the case $k=4, \ell=3$. Moreover, for $k=3$, by fixing $\left|U_{j}\right|=3$ for all $j$, the proofs of both lemmas, Lemma 4.1 and Lemma 4.2, go through and yield that $\operatorname{sat}(3 n, 3,2)=\Theta\left(n^{2}\right)$. As we mentioned in the Introduction, it has been proved in [18], via a different construction, that $\operatorname{sat}(n, k, k-1)=\Theta\left(n^{k-1}\right)$ for all $k \geqslant 3$.

A big open problem is to extend our result to all $1 \leqslant \ell \leqslant k-1$, that is, to prove the following conjecture.

Conjecture 5.1. For all $1 \leqslant \ell \leqslant k-1, k \geqslant 2$, sat $(n, k, \ell)=\Theta\left(n^{\ell}\right)$.

The smallest open case is $k=4, \ell=2$.

\section{Acknowledgements}

We thank the reviewer for carefully reading our manuscript and for giving detailed comments and suggestions that have been helpful to improve the manuscript.

\section{References}

[1] C. Berge. Nombres de coloration de l'hypergraphe $h$-parti complet. Springer Lecture Notes in Math., 411:13-20, 1975.

[2] J. A. Bondy. Variations on the hamiltonian theme. Canad. Math. Bull., 15:57-62, 1972.

[3] L. Clark, R. Crane, R. Entringer and H. Shapiro. On smallest maximally nonHamiltonian graphs. Proceedings 17th Southeastern international conference on combinatorics, graph theory, and computing (Boca Raton, Fla., 1986), 53:215-220, 1986.

[4] L. Clark and R. Entringer. Smallest maximally non-Hamiltonian graphs. Period. Math. Hungar., 14(1):57-68, 1983.

[5] L. H. Clark, R. C. Entringer and H. D. Shapiro. Smallest maximally nonhamiltonian graphs II. Graphs Combin., 8:225-231, 1992. 
[6] Andrzej Dudek and A. Frieze. Loose Hamilton Cycles in Random Uniform Hypergraphs. Electron. J. Combin., 18:P48, 2011.

[7] Andrzej Dudek and A. Frieze. Tight Hamilton cycles in random uniform hypergraphs. Random Structures Algorithms, 42(3):374-385, 2013.

[8] Aneta Dudek, G. Y. Katona and A. Żak. Hamilton-chain saturated hypergraphs. Discrete Math., 310:1172-1176, 2010.

[9] Aneta Dudek and A. Żak. On Hamilton-chain saturated uniform hypergraphs. Discrete Math. Theor. Comput. Sci., 14:21-28, 2012.

[10] A. Frieze. Loose Hamilton Cycles in Random 3-Uniform Hypergraphs. Electron. J. Combin., 17:N28, 2010.

[11] R. Glebov, Y. Person and W. Weps. On extremal hypergraphs for Hamiltonian cycles. European J. Combin., 33:544-555, 2012.

[12] A. Gyárfás, G. Sárközy and E. Szemerédi. Monochromatic Hamiltonian 3-tight Berge cycles in 2-colored 4-uniform hypergraphs. J. Graph Theory, 63:288-299, 2010.

[13] P. Haxell, T. Łuczak, Y. Peng, V. Rödl, A. Ruciński, M. Simonovits and J. Skokan. The Ramsey number for hypergraph cycles I. J. Combin. Theory Ser. A, 113:67-83, 2006.

[14] P. Haxell, T. Łuczak, Y. Peng, V. Rödl, A. Ruciński and J. Skokan. The Ramsey number for 3-uniform tight hypergraph cycles. Combin. Probab. Comput., 18:165204, 2009.

[15] G. Y. Katona. Hamiltonian chains in hypergraphs, a survey. Graphs, Combinatorics, Algorithms and its Applications, (ed. S. Arumugam, B. D. Acharya, S. B. Rao), Narosa Publishing House, 2004.

[16] G. Y. Katona and H. Kierstead. Hamiltonian chains in hypergraphs. J. Graph Theory, 30:205-212, 1999.

[17] V. Rödl and A. Ruciński. Dirac-type questions for hypergraphs - a survey (or more problems for Endre to solve). Bolyai Soc. Math. Studies 21 (2010), An Irregular Mind (Szemerédi is 70), Bolyai Soc. Math. Studies 21:561-590, 2010.

[18] A. Żak. Growth order for the size of smallest hamiltonian chain saturated uniform hypergraphs. European J. Combin., 34:724-735, 2013. 